\title{
SMC approves nine new medicines for NHS Scotland
}

The Scottish Medicines Consortium (SMC) has published advice on nine new medicines for use by NHS Scotland.

Firstly, following assessment within an ultra-orphan pathway introduced last year, the SMC has published an initial assessment report on the use of cerliponase- $\alpha$ [Brineura] in children with neuronal ceroid lipofuscinosis type 2. Cerliponase- $\alpha$ may improve quality of life for patients and their families by stabilising this inherited, life-limiting condition that leads to progressive brain damage. The treatment will be available for use by NHS Scotland for a 3-year period whilst information is gathered by the submitting pharmaceutical company to provide a required plan detailing how further data on the treatment effects will be collected. Following this, the evidence will be reviewed by SMC and a decision made about routine availability in NHS Scotland.

Secondly, ibrutinib [Imbruvica] was accepted for the treatment of Waldenstrom's macroglobulinaemia when given in combination with rituximab. Ibrutinib, the first treatment to be specifically licenced for this condition, can improve progression-free survival and was considered via SMC's Patient and Clinician Engagement (PACE) process. Also accepted through the PACE process were carfilzomib [Kyprolis] for multiple myeloma; lenalidomide [Revlimid] as a maintenance treatment for myeloma in stem cell transplant recipients; and avelumab [Bavencio] for advanced renal cell carcinoma when given in combination with axitinib.

Siponimod [Mayzent] was accepted for the treatment of people with active secondary progressive multiple sclerosis, making it the first disease modifying therapy for this patient group. Lastly, via an expedited approach aimed at minimising delays to patient access during the early stage of the COVID-19 pandemic, the SMC accepted lenalidomide [Revlimid] as combination treatment with rituximab for adults with previously treated follicular lymphoma; meropenem/vaborbactam [Vaborem] for multidrug-resistant serious infections; and budesonide [Jorveza] for eosinophilic esophagitis in adults.

Scottish Medicines Consortium. October 2020 decisions news release. Internet Document : 12 Oct 2020. Available from: URL: https://www.scottishmedicines.org.uk/about-us/ latest-updates/october-2020-decisions-news-release/ 